\title{
AN ESSAY ON TERMINOLOGY, MYTHS, - AND KNOWN \\ FACTS: SOLAR TRANSIENT - FLARE - CME - DRIVER \\ GAS - PISTON - BDE - MAGNETIC CLOUD - SHOCK WAVE \\ - GEOMAGNETIC STORM
}

\author{
RAINER SCHWENN \\ Max Planck Institut fr Aeronomie, D 37191 Katlenburg-Lindau, Germany
}

\begin{abstract}
In the field of solar-terrestrial relations a clear and unique terminology is needed in order to abolish and avoid unnecessary confusion between the scientists from several involved disciplines. For example, the widely used abbreviation CME (for coronal mass ejection) has turned out to be somewhat misleading. Early on it had been known that other than coronal material is often involved in such events. The discoverers observed transient events of mass ejections from the sun, which could be observed in the corona owing to the newly available coronagraphs. This article is meant to clarify the terminology, with emphasis on giving credit to the original discoverers and the terms they introduced. With this aim in mind I suggest some minor modifications of the terminology.
\end{abstract}

Key words: solar-terrestrial, coronal mass ejection, coronagraphs

\section{Introduction}

With the advent of earth-orbiting white light coronagraphs in the early $1970 \mathrm{~s}$ could the existence of transient mass ejections from the sun - in addition to the continuously blowing solar wind - be firmly established. The various authors used various terms for the phenomena they discovered. These terms were originally fairly descriptive, and no new names or abbreviations were created for several years. It was not before 1982 that the abbreviation "CME" (for "coronal mass ejection") appeared in the literature. This term has made its way in that it became soon a synonym in its own right, and is now being used widely and readily. One is indeed tempted to believe that this phenomenon is much better understood than it actually is. Some people no longer reflect upon its original meaning and often use the term in a misleading way.

Agreeing on an exact nomenclature has not been considered a crucial issue for a long time, since the concerned community (in coronal and interplanetary plasma physics) used to know pretty well what was meant. However, recently the significance of coronal dynamic processes has begun leaking through to neighboring communities: on the one side, classical solar physics got to realize that "their" energetic solar transient events like flares are intimately related (maybe even triggered and energized) by processes in the corona above. On the other side, researchers in magnetospheric physics and geophysics had to accept that "their" strong geomagnetic storms are more 
closely associated with mass ejections at the sun rather than with solar flares.

With so many scientists from several disciplines involved in disentangling the long chain of actions and reactions between sun and earth, we need a clear and unique terminology in order to abolish and avoid unnecessary confusion. This article is meant to help in this respect, with emphasis on giving credit to the original discoverers and the terms they introduced. Also, I offer some minor modifications of the terminology presently being used.

Revisiting all the work done in context with solar transient activity is a very rewarding exercise. I went through it in pretty much detail and listed my findings and all the relevant literature in an extended version of this paper (to be submitted to Space. Sci. Rev.). In the present summary I constrain myself to the major issues and key references.

\section{Observations, interpretations, and terminology}

It all started precisely on September 1st, 1859, when Carrington happened to observe on the sun a sudden short-lived breakout of intense white light (see Meadows, 1970). This first noted observation of a solar flare was followed about 17 hours later by an intense geomagnetic storm on earth. Carrington noticed and reported the apparent association. "As the century progressed, the belief that major terrestrial magnetic fluctuations were specifically connected with chromospheric flares received growing support", as Meadows (1970) wrote.

There is no doubt that this was the beginning of both the research on what we now call solar terrestrial relations in general, and the flaregeomagnetism association in particular. This latter issue has recently turned into a matter of heavy debate. On one side we find mainly geophysicists who have been using this association traditionally for their studies of geomagnetic activity. On the other side there are the plasma physicists studying the corona and heliosphere who claim that the real association is found with mass ejection events on the sun and the shock waves they produce, and that flares may or may not occur in loose context with them. In this ongoing debate the one side accuses the other of adhering to a "solar flare myth" (Gosling, 1993). Vice versa this side is reproached of not realizing what a flare really is and of replacing an old paradigm with a new one. After all, it is this controversy that led me to write this "essay".

With the discovery of the cosmic radiation in the early 20 th century, the solar-connected variations of cosmic ray intensity became a subject of intense research. Morrison (1954) thought of the "emission from the active sun of diffuse clouds of ionized hydrogen bearing a turbulent magnetic field". This new concept of magnetized clouds was then promoted by many authors, e.g., 
by Gold $(1955,1959)$. He argued that the well-known sudden commencement of a geomagnetic storm can only be explained in terms of the arrival of a highly supersonic shock wave which he thought is driven by a magnetized cloud ejected from the sun.

Many pieces of evidence for the actual occurrence of transient ejection processes on the sun were found both from solar radio observations (Type IV radio bursts as indicators of ejected plasmoids, Boischot, 1957), and in situ from space probes. Soon after the experimental verification of the existence of a continuously blowing solar wind in 1962, the occurrence of transient interplanetary shock waves was also confirmed (Sonett et al., 1964). Around 1968 evidence was found that some shocks are apparently followed by a different type of plasma characterized by an unusually high helium content (Hirshberg et al., 1970). The driver gas or shock piston concept came up.

With the arrival of space-borne coronagraphs on OSO 7 and Skylab (see, e.g., Tousey, 1973, and Gosling et al., 1974) the ejection of huge amounts of mass from the sun could be made visible in an impressive way. These photographs are indeed spectacular. The authors described their discoveries as plasma clouds, electron clouds, mass ejections from the sun, coronagraph observed mass ejections, solar mass ejections, mass ejection coronal transient, and the like.

Thousands of mass ejections were later on observed by the coronagraphs on the satellite P78-1 and the Solar Maximum Mission, and almost as many papers were written. A series of extensive reviews covers progress in this vivid field of research fairly well (see, e.g., (Gosling et al., 1976; MacQueen, 1980; Schwenn, 1986; Kahler, 1992). In the present review I will rather continue to restrict myself to those particular issues, where new features got new names.

Gosling et al. (1975) spoke of coronal mass ejection events. It is important to note that this new term (which was used here for the first time, I believe) was NOT supposed to infer an ejection of coronal mass. Early on, it had been clear that other than coronal material is often involved in such events (Hildner et al., 1975; Schwenn et al, 1980; Sheeley et al, 1981). What was rather meant is that there are events of mass ejections, and they can be observed in the corona owing to the newly available coronagraphs. Furthermore, the new term expresses its character as the designation of a process, rather than the product of such process, i.e. the "ejecta". This linguistic difference became less apparent when the shortened term coronal mass ejections and finally the abbreviation $C M E$ got into use.

For several years no unique nomenclature emerged. This could not be better illustrated than by the paper by Burlaga et al. (1978): they used the term CME for cold magnetic enhancements, i.e. something completely different! However, it was a similar group of authors (Burlaga et al., 1982) which finally introduced the acronym CME in its present meaning. 
Hundhausen et al. (1984) defined a CME "to be an observable change in coronal structure that (1) occurs on a time-scale between a few minutes and several hours and (2) involves the appearance of a new, discrete, bright, white-light feature in the coronagraph field of view". To be more specific, I would like to add "appearance and outward motion...". This definition is very fortunate, since it underlines the observational aspect, it stresses the transient event character, and it does not imply an interpretation of the feature and its potential origin. In particular, it does NOT infer any conjunction with "coronal mass", in contrast to what the term CME itself does. I prefer to speak of solar mass ejections according to Gosling's (1975) first use of the term, or $S M E s$ (if an abbreviation is considered necessary) rather than of CMEs. Note also that Hundhausen's definition (which applies to SMEs as well) restricts the applicability of the term to the sun's proximity where the phenomenon was actually discovered. In other words, it does NOT allow to call any cloud of strange material seen anywhere in interplanetary space a CME.

To this day, we do not understand the basic physics of SMEs, e.g., what causes them, how and where are the ejecta accelerated, what is the role of magnetic reconnection processes. Also, we do not know by which drastic transformations the coronagraph observed features at SMEs are turned into the plasma clouds encountered in situ. For example, the well-known threepart structure often seen at major SMEs (bright loop of coronal plasma, followed by dark void and finally by cold prominence material, see Illing and Hundhausen, 1985) has no counterpart discernible in interplanetary space. On the other hand, there are several marked signatures of driver gas clouds (see, e.g., Borrini et al., 1982; Gosling, 1990): high helium content, unusual ionization states, depressions of proton and electron temperatures, bi- directional streaming of suprathermal electrons and energetic protons. Usually only a small subset of these criteria is found. Some of the clouds show the signatures of magnetic clouds (Burlaga et al., 1982): characteristic rotation of the magnetic field vector, strong field with low variance, very low plasma beta. Others don't. At some shocks no driver gas or cloud signatures at all are found. In contrast, some other clouds are not associated with shocks. None of all the signatures has any equivalence in coronagraph pictures! Considering all this I prefer to speak of injected plasma clouds (IPCs), following Morrison's (1954) terminology. There is no doubt that IPCs are the product of SMEs, after all, but there are still many open questions about the physical processes involved.

When a plasma cloud is injected into the ambient solar wind at higher speed, the ambient magnetic field is deflected ("draped") around it, similarly to what occurs at the earth's magnetopause or at any other obstacle to the solar wind (McComas et al., 1987). Depending on the topology of both: the ambient field and the IPC driving the shock, large meridional 
field components may occur. In particular, in case of a southward pointing field reconnection processes at the earth's magnetopause may drive large non-recurrent geomagnetic storms, provided the earth is hit at all.

The associations of SMEs with other forms of solar activity on the one hand and with heliospheric or geophysical phenomena on the other hand has been studied in much detail. In their first paper Gosling et al. (1974) noted already that "18 of these (30) ejections were associated with active and eruptive prominences and surges: only 3 ejections appear to have been flare initiated". This surprisingly loose association between the two most energetic types of transient events on the sun was later on confirmed in several ways. Furthermore, in many cases with an apparent flare association the flare was found to begin AFTER the visible lift-off of the SME (Harrison et al., 1985). Quite logically, for those cases the flare could be ruled out as being the cause of the SME.

On the other hand, a very close association between SMEs and shocks was established (Sheeley et al., 1985): in case the position of an observing space probe is included within the angular span of a SME, the chances for the detection of an associated shock is almost $100 \%$ when the SME speed exceeds about $400 \mathrm{~km} / \mathrm{s}$. In contrast, the association between flares and shocks has always been imperfect. That suggests also that flares may be secondary effects of the shock generation process in the course of SMEs.

These observational facts have been found and published as early as 1985, and more evidence has been piled up in succeeding years. Quite evidently, they are in sharp conflict with the flare-geomagnetism concept that had been in place since Carrington's discovery 125 years ago. That is probably the reason why the new concept was not readily accepted, although it had been noticed that the old one was not very reliable if applied for prediction purposes. Only when Gosling (1993) pointed out rather drastically that the solar flare myth should finally be put to rest, this controversy became an issue of heated debates in the various scientific communities.

\section{Summary}

At this time, it appears useful to summarize the status of our present knowledge in a few short "safe" statements:

- There are transient solar mass ejection (SME) events occurring in various forms and at various speeds.

- Solar flares may or may not occur in loose context with SMEs, both spatially and timely.

- SME events produce ejecta, specifically: coronal plasma (at coronal temperatures, almost fully ionized), and occasionally prominence material (with significant amounts of "cold" gas at low ionization states) 
- Major fractions of the ejecta leave the sun for ever and propagate throughout the heliosphere as interplanetary plasma clouds (IPCs).

- Most IPCs leaving the sun with speeds of more than about $400 \mathrm{~km} / \mathrm{s}$ will eventually drive a large-scale shock wave ahead of themselves.

- Out of several potential signatures of IPCs usually only a subset does actually occur.

- There are shocks without a discernible IPC following them.

- There are IPCs discernible by some characteristic signatures which do not have an associated shock in front of them.

- Strong non-recurrent geomagnetic storms are initiated when the interplanetary magnetic field has a southward component due to draping by an IPC or the clouds internal magnetic cloud topology or both.

After all, there is now clear evidence that interplanetary shock waves and interplanetary plasma clouds are closely associated with solar mass ejections and that these plasma clouds are the interplanetary counterparts of the solar ejecta. However, the detailed chain of actions and reactions between the sun and the outer parts of the heliosphere remains to be disentangled, and we should be prepared for further surprises.

\section{References}

Boischot, A.: 1957, Comptes Rendus Acad. Sci., Paris 244, 1326.

Borrini, G., Gosling, J.T., Bame, S.J., and Feldman, W.C.: 1982, J. Geophys. Res. 87, 4365 .

Burlaga, L.F., Ness, N.F., Mariani, F., Bavassano, B., Villante, U., Rosenbauer, H., Schwenn, R., and Harvey, J.: 1978, J. Geophys. Res. 83, 5167.

Burlaga, L.F., Klein, L., Sheeley, N.R., Michels, D.J., Howard, R.A., Koomen, M.J., Schwenn, R., and Rosenbauer, H.: 1982, Geophys. Res. Lett. 9, 1317.

Gold, T.: 1955, Gas Dynamics of Cosmic Clouds, North-Holland Publ. Co., Amsterdam, 103.

Gold, T.: 1959, J. Geophys. Res. 64, 1665.

Gosling, J.T., Hildner, E., MacQueen, R.M., Munro, R.H., Poland, A.I., and Ross, C.L.: 1974, J. Geophys. Res., 79, 4581.

Gosling, J.T., Hildner, E., MacQueen, R.M., Munro, R.H., Poland, A.I., and Ross, C.L.: 1975, Solar Phys., 40, 439.

Gosling, J.T.: 1976, D.J. Williams (ed.), in Physics of Solar Planetary Environments 1, Boulder, published by AGU, p. 286.

Gosling, J.T.: 1990, Physics of Magnetic Flux Ropes, Geophysical Monograph 58, AGU Washington, 343.

Gosling, J.T.: 1993, J. Geophys. Res., 98, 18937.

Harrison, R.A., Waggett, P.W., Bentley, R.D., Phillips, K.J.H., Bruner, M., Dryer, M., and Simnett, G.M.: 1985, Solar Phys., 97, 387.

Hildner, E., Gosling, J.T., MacQueen, P.M., Munro, R.H., Poland, A.I., Ross, C.L.: 1975, Solar Phys., 42, 163.

Hirshberg, J., Alksne, A., Colburn, D.S., Bame, S.J., and Hundhausen A.J.: 1970, J. Geophys. Res., 75, 1.

Hundhausen, A.J., Sawyer, C.B., House, L.L., Illing, R.M.E., and Wagner, W.J.: 1984, J. Geophys. Res., 89, 2639.

Illing, R.M.E. and Hundhausen, A.J.: 1985, J. Geophys. Res., 90, 275. 
Kahler, S.W.: 1992, Ann. Rev. Astron. Astrophys., 30, 113.

MacQueen, R.M.: 1980, Phil. Trans. R. Soc., London, 297, 605.

McComas, D.J., Gosling, J.T., Winterhalter, D., and Smith, E.J.: 1987, J. Geophys. Res., 93, 2519.

Meadows, A.J.: 1970, Early Solar Physics, Pergamon Press, Oxford, England.

Morrison, P.: 1954, Phys. Rev., 95, 641.

Schwenn, R., Rosenbauer, H., Muser, K.-H.: 1980, Geophys. Res. Lett. 7, 201.

Schwenn, R.: 1986, Space Sci. Rev., 44, 139.

Sheeley, Jr., N.R., Michels, D.J., Howard, R.A., Koomen, M.J.: 1981, EOS Trans. AGU, $62,153$.

Sheeley Jr., N.R., Howard, R.A., Koomen, M.J., Michels, D.J., Schwenn, R., Muser, K.H., and Rosenbauer, H.: 1985a, J. Geophys. Res., 90, 163.

Sonett, C.P., Colburn, D.S., Davis, L., Smith, E.J., and Coleman, P.J.: 1964, Phys. Rev. Letters, 13, 153.

Tousey, R.: 1973, Space Res., 13, 713. 